\title{
Monitoring in neurointensive care - the challenge to detect delayed cerebral ischemia in high-grade aneurysmal SAH
}

\section{Asita S. Sarrafzadeh ${ }^{1,2 *}$, Peter Vajkoczy ${ }^{2}$, Philippe Bijlenga ${ }^{1}$ and Karl Schaller ${ }^{1}$}

' Division of Neurosurgery, Geneva Neuroscience Center, Faculty of Medicine, University of Geneva, Geneva, Switzerland

${ }^{2}$ Department of Neurosurgery, Charité - Universitätsmedizin Berlin, Berlin, Germany

\section{Edited by:}

Elham Rostami, Uppsala University Hospital, Sweden

\section{Reviewed by:}

Antonino F. Germano, University of Messina, Italy

Niklas Marklund, University of Uppsala, Sweden

Elisabeth Ronne Engström, Uppsala University Hospital, Sweden

\section{*Correspondence:}

Asita S. Sarrafzadeh, Division of Neurosurgery, Geneva University Hospitals, Rue Gabrielle Perret-Gentil

4, Geneva 1211, Switzerland

e-mail: asita.sarrafzadeh@hcuge.ch
Delayed cerebral ischemia (DCl) is a feared and significant medical complication following aneurysmal subarachnoid hemorrhage (aSAH). It occurs in about $30 \%$ of patients surviving the initial hemorrhage, mostly between days 4 and 10 after aSAH. Clinical deterioration attributable to $\mathrm{DCl}$ is a diagnosis of exclusion and especially difficult to diagnose in patients who are comatose or sedated. The latter are typically patients with a high grade on the World Federation of Neurosurgical Societies scale (WFNS grade 4-5), who represent approximately $40-70 \%$ of the patient population with ruptured aneurysms. In this group of patients, the incidence of $\mathrm{DCl}$ is often underestimated and higher when compared to low WFNS grade patients. To overcome difficulties in diagnosing $\mathrm{DCl}$, which is especially relevant in sedated and comatose patients, the article reports the most recent recommendation for definition of $\mathrm{DCl}$ and discusses their advantages and problematic issues in neurocritical care practice. Finally, appropriate neuromonitoring techniques and their clinical impact in high-grade SAH patients are summarized.

Keywords: delayed cerebral ischemia, vasospasm, subarachnoid hemorrhage, aneurysm, neuromonitoring

\section{INTRODUCTION: THE PROBLEM - DELAYED CEREBRAL ISCHEMIA}

Delayed cerebral ischemia (DCI) is a feared and significant medical complication following aneurysmal subarachnoid hemorrhage (aSAH). It occurs in about $30 \%$ of patients surviving the initial hemorrhage, mostly between days 4 and 10 after aSAH. The known clinical symptoms such as decrease in the level of consciousness and focal signs such as aphasia and hemiparesis may be reversible or otherwise progress to cerebral infarction resulting in an unfavorable outcome or even death (1). Clinical deterioration attributable to DCI is a diagnosis after exclusion of other causes (such as infection, hypotension, hyponatremia, and others), and it is especially difficult to diagnose in patients who are comatose or sedated $(1,2)$. The latter are typically patients with a high grade on the World Federation of Neurosurgical Societies scale (3) (WFNS grade 4-5), which represent approximately 40$70 \%$ of the patient population with ruptured aneurysms. In this group of patients, the incidence of DCI is often underestimated (4) and higher when compared to low WFNS grade patients (5). As shown by Rabinstein and colleagues, as independent predictive factors of a very low risk to develop DCI at age 68 years or older, WFNS I-III at presentation and a modified Fisher grade 1-2 were identified, with specificity of $100 \%$ and positive predictive value of $100 \%$ (5). As mortality for high-grade patients remains high, efforts have been made to improve diagnosis and treatment of DCI-related damage and to identify a patient subgroup who can expect a better outcome (6).

\section{AND ANGIOGRAPHIC VASOSPASM}

Angiographic vasospasm can be associated with reductions in cerebral perfusion measured with 15O-positron emission tomographic imaging (7). However, regional hypoperfusion and oligemia frequently occurred in territories and patients with no angiographic vasospasm. Furthermore, the development of arterial spasm as diagnosed by angiography or transcranial Doppler (TCD) had no consistent relationship with 3-month outcome (8). Other factors in addition to large-vessel narrowing must contribute to critical reductions in perfusion. So far, because of the common association between clinical deterioration from DCI and angiographic vasospasm, arterial narrowing on angiography and increased blood flow velocities on TCD ultrasound examination are often used as surrogate diagnostic tool (2). This, however, is problematic as the old paradigm of "clinical deterioration due to DCI is equivalent to angiographic vasospasm" has changed in the last years (9). One of the landmarks was the clazosentan-study results (conscious-2), an endothelin-receptor antagonist that effectively resolves angiographic vasospasm, but failed to reduce mortality, DCI-related morbidity, or functional outcome in relation to aSAH (10). Patients with aSAH may have clinical deterioration attributable to DCI in the absence of radiologically confirmed vasospasm $(11,12)$. This may be the case because angiography is not obtained at the moment of clinical deterioration or because DCI is caused by other factors (2).

\section{AND INFARCTION - DEFINITIONS}

Throughout the literature, various means of defining vasospasm are used. To overcome difficulties to diagnose DCI, which is especially relevant in sedated and comatose patients, an excellent summary of possible definitions and their clinical impact, evaluated in 580 aSAH patients, was performed by Frontera and colleagues (8). More recently, to improve comparability of future SAH studies, 
two different definitions were reformulated by a multidisciplinary research group (2): (1) clinical deterioration caused by DCI and (2) cerebral infarction.

Delayed cerebral ischemia is defined as "the occurrence of focal neurological impairing (such as hemiparesis, aphasia, apraxia, hemianopsia, or neglect) or a decrease of at least two points on the Glasgow Coma Scale [either on the total score or one of its individual components (eye, motor on either side, verbal)]. This should last for at least $1 \mathrm{~h}$, and is not apparent immediately after aneurysm occlusion and cannot be attributed to other causes by means of clinical assessments, cerebral CT or MRI scanning, and appropriate laboratory studies. Nevertheless, the diagnosis of DCI remains fairly subjective.

Cerebral infarction is defined as presence of cerebral infarction on CT or MRI scan of the brain within 6 weeks after SAH, or on the latest CT or MRI scan made before death within 6 weeks, or proven at autopsy, not present on the CT or MR scan between 24 and $48 \mathrm{~h}$ after early aneurysm occlusion, and not attributable to other causes (such as clipping or endovascular treatment). Hypodensities on CT imaging resulting from ventricular catheter or intraparenchymal hematoma should not be regarded as cerebral infarctions from DCI.

To remember, cerebral infarction correlates with the territory of angiographic vasospasm in only $25-81 \%$ of SAH patients (1, 13). The term "vasospasm" was recommended to be reserved for angiographic arterial narrowing. The occurrence of DCI should be described separately from the results of angiography as not all patients who experience DCI have angiographic vasospasm, and not all patients with angiographic spasm have DCI.

\section{REAL LIFE IN NEUROINTENSIVE CARE}

Though knowing that the simple association of radiographic evidence of vasospasm with clinical features of cerebral ischemia does not take into account the different factors that may contribute to DCI, most neurointensivist know a series of cases showing an excellent correlation between an observed clinical deterioration and angiographic narrowing, with deficits easily reversible with an increase of mean arterial blood pressure. In our view, these concern mainly low WFNS grade patients, while in highWFNS grade patients, the large spectrum of difficult-to-diagnose $\mathrm{DCI} /$ infarction is common. The diagnosis of DCI is limited and the diagnosis of cerebral infarction possible only retrospectively once an infarct is found radiographically (8). Obviously, interobserver agreement rate is higher for cerebral infarction and easier to evaluate in high-WFNS grade patients. Nevertheless, a sophisticated evaluation is relevant also in this "difficult to evaluate and treat" patient group, e.g., the clinical course is often complicated by myocardial dysfunction, pneumonia, and acute respiratory distress syndrome secondary to the aneurysm ruptures that further complicate the "vasospasm" treatment and participates in the worst outcome of high WFNS. Some of the factors proceeding to infarction might be reversible if treated. The "infarction diagnosis" reflects an under treatment of ongoing not detected ischemia.

There are a series of worth-to-measure techniques, some of them shortly summarized in the following, aimed to detect "asymptomatic" ischemia in high-WFNS grade patients.

\section{POSSIBLE CAUSES OF DCI}

There are other potential mechanisms of primary and secondary brain injury events related to DCI (14), some of them detectable by neuromonitoring techniques.

(1) Intracranial pressure (ICP) and cerebral perfusion pressure (CPP): ICP and CPP monitoring, obviously important components of neurocritical care, are widely used in comatose aSAH patients. ICP is preferentially measured as external ventricular drainage (EVD), while in absence of hydrocephalus/slit ventricles, an ICP probe may be used instead, allowing continuous CPP monitoring. A high ICP ( $>20 \mathrm{mmHg}$ ) is considered as an indirect but unspecific warning sign, is associated with energy failure, high secretion of pro-inflammatory interleukins, and possibly aggravates vasospasm. Optimal CPP is targeted individually depending on several factors (ruptured aneurysm treated and presence of DCI). Since CPP might not be available in all patients, current guidelines (15) recommend (A) maintenance of euvolemia and normal circulating blood volume to prevent DCI (Class I; Level of Evidence B, revised recommendation from previous guidelines); (B) (new recommendation) induction of hypertension for patients with DCI unless blood pressure is elevated at baseline or cardiac status precludes it (Class I; Level of Evidence B); and (C) prophylactic hypervolemia before the development of angiographic spasm is not recommended (Class III; Level of Evidence B).

(2) Among other pathomechanisms proposed, ischemia-producing cortical spreading depolarizations (CSDs) are likely to be involved in DCI development (16). CSDs are monitored by use of subdural electrodes inserted after aneurysm clipping or an EVD. CSD are self-propagating tissue depolarizations, associated with cessation of synaptic activity, surges of extracellular potassium, opening of the blood-brain barrier with edema formation, tissue hypoxia, and inversion to the normal CBF response to neuroglial activity $(11,17)$. Recent clinical data confirm that a complex cascade of interconnected molecular, metabolic, electrophysiological, and vascular disturbances may lead to ischemia, homologous to animal models (18). The discovery of SDs may lead to a better explanation of DCI in patients with SAH. However, the definite role of SD and its therapeutic implications in SAH are yet unclear (19). Results from the ongoing multicenter Discharge-1 trial (Depolarizations in ISCHaemia after subARachnoid haemorrhaGE-1) are awaited.

(3) Disturbed cerebrovascular autoregulation in the first 5 days after SAH significantly increases the odds of DCI occurrence and was shown to have a better predictive value than classical TCD monitoring protocols (20). Initial (first $48 \mathrm{~h}$ ) preserved cerebrovascular autoregulation in severe SAH grade patients is a good predictor of survival. Optimal CPP (CPPopt) is defined as the lowest CPP corresponding to the strongest cerebrovascular autoregulation activity. It has been shown that CPPopt increases during the vasospasm period for almost all SAH patients but more significantly in the group of patients who suffer vasospasm as assessed by TCDs (21). 
(4) Neurological wake-up test (interruption of sedation, IS-trial). Clinical deteriorations, such as occurrence of focal neurological impairing such as aphasia and hemiparesis are warning signs for potentially DCI, which are difficult to evaluate in high-grade SAH patients. The effect of interruption of sedatives and analgesics on neurologic assessment, hemodynamic changes, brain metabolism, and brain tissue oxygen have shown that (1) IS-trials were not attempted on one-third of eligible days due to safety concerns, and that (2) one-third of performed trials had to be stopped due to a critical increase in ICP and impending brain tissue hypoxia. All IS-trials were associated with cardiopulmonary stress, and detection of a new neurological deficit that led to changes in management occurred in only one trial (22). Nevertheless, cerebral metabolism and oxygenation was not affected in a recent trial (traumatic brain injury, TBI, patients) (23). This suggests that IS-trials are safe in the majority of patients but that the test should be individualized and avoided in patients reacting with markedly increased ICP and/or decreased CPP (23). In contrast to TBI, the dynamics for detection of a new deficit in aSAH patients are more variable and potentially treatable, suggesting an important role for IS-trials in aSAH patients. Lacking detailed guidelines, the limitation for this maneuver, in our view, is the stability of ICP and the targeted CPP avoiding arterial hypotension/cerebral hypoperfusion and obviously, ideally under control of further neuromonitoring parameters.

(5) Monitoring of cerebral oxygenation. The regionally monitoring brain tissue partial pressure of oxygen sensor and the globally measuring jugular bulb oximetry allowing further analysis of arterial-venous differences of energy-related factors are commonly used techniques for detection of cerebral hypoxia/ischemia. A decrease of brain tissue $\mathrm{PO}_{2}$ $(<15-10 \mathrm{mmHg})$ and/or jugular bulb oxygenation $(<70 \%)$ are warning signs and sensibilize the neurointensivist for occurring processes leading to progressive, clinically not detectable ischemia, infarction, and unfavorable outcome in this high-risk SAH population.

(6) Cerebral metabolism. Lacking clinical features to diagnose a neurological deterioration in high-WFNS grade patients, cerebral microdialysis provides information on the metabolic state of the injured brain and allows for determination of a variety of markers of ischemia and immunocompetence in the cerebral extracellular fluid (ECF). This is of relevance since both the blood-brain and blood-CSF barriers may be variably disrupted following aSAH, rendering blood and CSF levels of such markers less reliable. Probes are implanted directly into the region of interest, typically after aneurysm clipping. Profound changes in extracellular glucose, lactate, glutamate, and glycerol in patients with signs of acute or delayed ischemia have been shown $(24,25)$. Accepted thresholds to indicate metabolic crisis/ischemia are in the first line defined for the lactate-pyruvate ratio $>25-40$ and glucose $<0.5 \mathrm{mM}$ and in second line for glutamate $>40 \mathrm{mM}$; however, individual trends are very important for careful interpretation. The method was especially valuable in patients with acute focal deficits on admission (AFND) due to concomitant intracerebral hemorrhage or edema post aSAH $(26,27)$. As it is a time- and cost-intense method, only few centers continued monitoring, mainly focusing on possible biomarkers predicting DCI, early brain injury (28), and related inflammatory processes (29).

\section{EARLY BRAIN INJURY, DCI, AND IMMUNE SYSTEM}

The finding that prevention of delayed vasospasm does not improve outcome in aSAH patients has brought into focus the influence of early brain injury on outcome of aSAH. A substantial amount of evidence indicates that brain injury begins at the aneurysm rupture, evolves with time, and plays an important role in patients' outcome. It is postulated that intraparenchymal activation of the immune system after aSAH contributes to secondary brain injury. Intraparenchymal inflammation has been linked to progression of aSAH-induced secondary brain damage $(30,31)$. More specifically, leukocyte-endothelial cell interactions appear to play a critical role in vasospasm. It is hypothesized that the development of DCI is promoted by these inflammatory processes leading to a decreased immunocompetence and secondarily to infectious complications (32). Putative promising markers to indicate immunosuppression are low HLADR and high interleukin-6 levels (thresholds depending on laboratory).

Another point is the entity of patients "at high risk" presenting with acute focal neurological deficits (AFND) on admission (26). Most, but not all of them, are high-WFNS grade patients; however, presenting early brain damages, such as an intracerebral hemorrhage, edema, and high ICP not related to hydrocephalus. These patients show the most severe immunodepression and highest infection rate of the aSAH population $(28,32)$.

\section{SUMMARY}

In conclusion, the use of clear definitions to classify: (1) occurring deficits such as AFND and DCI, (2) pathomechanisms such as CSD, cerebral hypoxia, disturbed cerebral autoregulation, cerebral metabolism, and inflammation alterations, and (3) resulting lesion using precise time windows and assessment techniques will allow to better share experiences on the disease and improve its understanding. Advanced neuromonitoring techniques may offer the neurointensivists complementary tools to better perceive the pathological condition and engage appropriate treatments that would otherwise lead to progressive, clinically not detectable ischemia, infarction, and unfavorable outcome in high-risk $\mathrm{SAH}$ population.

\section{REFERENCES}

1. Rabinstein AA, Friedman JA, Weigand SD, Mcclelland RL, Fulgham JR, Manno EM, et al. Predictors of cerebral infarction in aneurysmal subarachnoid hemorrhage. Stroke (2004) 35:1862-6. doi:10.1161/01.STR.0000133132.76983.8e

2. Vergouwen MD, Vermeulen M, van Gijn J, Rinkel GJ, Wijdicks EF, Muizelaar JP, et al. Definition of delayed cerebral ischemia after aneurysmal subarachnoid hemorrhage as an outcome event in clinical trials and observational studies: proposal of a multidisciplinary research group. Stroke (2010) 41:2391-5. doi:10.1161/STROKEAHA.110.589275

3. Teasdale GM, Drake CG, Hunt W, Kassell N, Sano K, Pertuiset B, et al. A universal subarachnoid hemorrhage scale: report of a committee of the World Federation of Neurosurgical Societies. J Neurol Neurosurg Psychiatry (1988) 51:1457. doi:10.1136/jnnp.51.11.1457

4. Schmidt JM, Wartenberg KE, Fernandez A, Claassen J, Rincon F, Ostapkovich $\mathrm{ND}$, et al. Frequency and clinical impact of asymptomatic cerebral infarction due 
to vasospasm after subarachnoid hemorrhage. J Neurosurg (2008) 109:1052-9. doi:10.3171/JNS.2008.109.12.1052

5. Crobeddu E, Mittal MK, Dupont S, Wijdicks EF, Lanzino G, Rabinstein AA. Predicting the lack of development of delayed cerebral ischemia after aneurysmal subarachnoid hemorrhage. Stroke (2012) 43:697-701. doi:10.1161/ STROKEAHA.111.638403

6. Hutchinson PJ, Power DM, Tripathi P, Kirkpatrick PJ. Outcome from poor grade aneurysmal subarachnoid haemorrhage - which poor grade subarachnoid haemorrhage patients benefit from aneurysm clipping? Br J Neurosurg (2000) 14:105-9. doi:10.1080/02688690050004516

7. Dhar R, Scalfani MT, Blackburn S, Zazulia AR, Videen T, Diringer M. Relationship between angiographic vasospasm and regional hypoperfusion in aneurysmal subarachnoid hemorrhage. Stroke (2012) 43:1788-94. doi:10.1161/ STROKEAHA.111.646836

8. Frontera JA, Fernandez A, Schmidt JM, Claassen J, Wartenberg KE, Badjatia N, et al. Defining vasospasm after subarachnoid hemorrhage: what is the most clinically relevant definition? Stroke (2009) 40:1963-8. doi:10.1161/STROKEAHA. 108.544700

9. Pluta RM, Hansen-Schwartz J, Dreier J, Vajkoczy P, Macdonald RL, Nishizawa S, et al. Cerebral vasospasm following subarachnoid hemorrhage: time for a new world of thought. Neurol Res (2009) 31:151-8. doi:10.1179/174313209X393564

10. Macdonald RL, Higashida RT, Keller E, Mayer SA, Molyneux A, Raabe A, et al. Clazosentan, an endothelin receptor antagonist, in patients with aneurysmal subarachnoid haemorrhage undergoing surgical clipping: a randomised, double-blind, placebo-controlled phase 3 trial (CONSCIOUS-2). Lancet Neurol (2011) 10:618-25. doi:10.1016/S1474-4422(11)70108-9

11. Ostergaard L, Aamand R, Karabegovic S, Tietze A, Blicher JU, Mikkelsen IK, et al. The role of the microcirculation in delayed cerebral ischemia and chronic degenerative changes after subarachnoid hemorrhage. J Cereb Blood Flow Metab (2013) 33:1825-37. doi:10.1038/jcbfm.2013.173

12. Woitzik J, Dreier JP, Hecht N, Fiss I, Sandow N, Major S, et al. Delayed cerebral ischemia and spreading depolarization in absence of angiographic vasospasm after subarachnoid hemorrhage. J Cereb Blood Flow Metab (2012) 32:203-12. doi:10.1038/jcbfm.2011.169

13. Weidauer S, Lanfermann H, Raabe A, Zanella F, Seifert V, Beck J. Impairment of cerebral perfusion and infarct patterns attributable to vasospasm after aneurysmal subarachnoid hemorrhage: a prospective MRI and DSA study. Stroke (2007) 38:1831-6. doi:10.1161/STROKEAHA.106.477976

14. Sabri M, Lass E, Macdonald RL. Early brain injury: a common mechanism in subarachnoid hemorrhage and global cerebral ischemia. Stroke Res Treat (2013) 2013:394036. doi:10.1155/2013/394036

15. Sander Connolly E, Rabinstein AA, Carhuapoma JC, Derdeyn CP, Dion J, Higashida RT, et al. Guidelines for the management of aneurysmal subarachnoid hemorrhage: a guideline. Stroke (2012) 43:1711-37. doi:10.1161/STR. 0b013e3182587839

16. Dreier JP. The role of spreading depression, spreading depolarization and spreading ischemia in neurological disease. Nat Med (2011) 17:439-47. doi: $10.1038 / \mathrm{nm} .2333$

17. Dreier JP, Major S, Pannek HW, Woitzik J, Scheel M, Wiesenthal D, et al. Spreading convulsions, spreading depolarization and epileptogenesis in human cerebral cortex. Brain (2012) 135:259-75. doi:10.1093/brain/awr303

18. Sakowitz OW, Santos E, Nagel A, Krajewski KL, Hertle DN, Vajkoczy P, et al. Clusters of spreading depolarizations are associated with disturbed cerebral metabolism in patients with aneurysmal subarachnoid hemorrhage. Stroke (2013) 44:220-3. doi:10.1161/STROKEAHA.112.672352

19. Sanchez-Porras R, Zheng Z, Santos E, Scholl M, Unterberg AW, Sakowitz OW. The role of spreading depolarization in subarachnoid hemorrhage. Eur J Neurol (2013) 20:1121-7. doi:10.1111/ene.12139

20. Budohoski KP, Czosnyka M, Smielewski P, Kasprowicz M, Helmy A, Bulters D, et al. Impairment of cerebral autoregulation predicts delayed cerebral ischemia after subarachnoid hemorrhage: a prospective observational study. Stroke (2012) 43:3230-7. doi:10.1161/STROKEAHA.112.669788
21. Bijlenga P, Czosnyka M, Budohoski KP, Soehle M, Pickard JD, Kirkpatrick PJ, et al. "Optimal cerebral perfusion pressure" in poor grade patients after subarachnoid hemorrhage. Neurocrit Care (2010) 13:17-23. doi:10.1007/s12028010-9362-1

22. Helbok R, Kurtz P, Schmidt JM, Stuart MR, Fernandez L, Connolly SE, et al. Effects of the neurological wake-up test on clinical examination, intracranial pressure, brain metabolism and brain tissue oxygenation in severely braininjured patients. Crit Care (2012) 16:R226. doi:10.1186/cc11880

23. Skoglund K, Hillered L, Purins K, Tsitsopoulos PP, Flygt J, Engquist H, et al. The neurological wake-up test does not alter cerebral energy metabolism and oxygenation in patients with severe traumatic brain injury. Neurocrit Care (2014) 20(3):413-26. doi:10.1007/s12028-013-9876-4

24. Nilsson OG, Brandt L, Ungerstedt U, Saveland H. Bedside detection of brain ischemia using intracerebral microdialysis: subarachnoid hemorrhage and delayed ischemic deterioration. Neurosurgery (1999) 45:1176-84; discussion 1184-5. doi:10.1097/00006123-199911000-00032

25. Sarrafzadeh A, Haux D, Plotkin M, Ludemann L, Amthauer H, Unterberg A. Bedside microdialysis reflects dysfunction of cerebral energy metabolism in patients with aneurysmal subarachnoid hemorrhage as confirmed by $15 \mathrm{O}-\mathrm{H} 2$ O-PET and 18 F-FDG-PET. J Neuroradiol (2005) 32:348-51. doi:10.1016/S01509861(05)83168-2

26. Sarrafzadeh A, Haux D, Sakowitz O, Benndorf G, Herzog H, Kuechler I, et al. Acute focal neurological deficits in aneurysmal subarachnoid hemorrhage: relation of clinical course, CT findings, and metabolite abnormalities monitored with bedside microdialysis. Stroke (2003) 34:1382-8. doi:10.1161/01.STR. 0000074036.97859 .02

27. Sarrafzadeh A, Haux D, Kuchler I, Lanksch WR, Unterberg AW. Poor-grade aneurysmal subarachnoid hemorrhage: relationship of cerebral metabolism to outcome. J Neurosurg (2004) 100:400-6. doi:10.3171/jns.2004.100.3.0400

28. Radolf S, Smoll N, Drenckhahn C, Dreier JP, Vajkoczy P, Sarrafzadeh AS. Cerebral lactate correlates with early onset pneumonia after aneurysmal SAH. Transl Stroke Res (2014) 5:278-85. doi:10.1007/s12975-013-0292-z

29. Sarrafzadeh A, Santos E, Wiesenthal D, Martus P, Vajkoczy P, Oehmchen M, et al. Cerebral glucose and spreading depolarization in patients with aneurysmal subarachnoid hemorrhage. Acta Neurochir Suppl (2013) 115:143-7. doi:10.1007/ 978-3-7091-1192-5_28

30. Sehba FA, Pluta RM, Zhang JH. Metamorphosis of subarachnoid hemorrhage research: from delayed vasospasm to early brain injury. Mol Neurobiol (2011) 43:27-40. doi:10.1007/s12035-010-8155-z

31. Sehba FA, Bederson JB. Nitric oxide in early brain injury after subarachnoid hemorrhage. Acta Neurochir Suppl (2011) 110:99-103. doi:10.1007/978-37091-0353-1_18

32. Sarrafzadeh A, Schlenk F, Meisel A, Dreier J, Vajkoczy P, Meisel C. Immunodepression after aneurysmal subarachnoid hemorrhage. Stroke (2011) 42:53-8. doi:10.1161/STROKEAHA.110.594705

Conflict of Interest Statement: The authors declare that the research was conducted in the absence of any commercial or financial relationships that could be construed as a potential conflict of interest.

Received: 31 March 2014; accepted: 04 July 2014; published online: 21 July 2014. Citation: Sarrafzadeh AS, Vajkoczy P, Bijlenga P and Schaller K (2014) Monitoring in neurointensive care - the challenge to detect delayed cerebral ischemia in high-grade aneurysmal SAH. Front. Neurol. 5:134. doi: 10.3389/fneur.2014.00134

This article was submitted to Neurotrauma, a section of the journal Frontiers in Neurology.

Copyright $\odot 2014$ Sarrafzadeh, Vajkoczy, Bijlenga and Schaller. This is an open-access article distributed under the terms of the Creative Commons Attribution License (CC $B Y)$. The use, distribution or reproduction in other forums is permitted, provided the original author(s) or licensor are credited and that the original publication in this journal is cited, in accordance with accepted academic practice. No use, distribution or reproduction is permitted which does not comply with these terms. 